\title{
Are you afraid of taking an online foreign language test?
}

Jesus Garcia Laborda a *,

Valencia Robles ${ }^{b}$,

Suggested Citation:

Abstract 
1. Introduction

2. Method

2.1. Research questions

3. Results 
4. Analysis

5. Conclusions 